\title{
Approximate non-destructive quantification of porphyrins in butter by front face fluorescence spectroscopy*
}

\author{
J.P. Wold ${ }^{1}$ and F. Lundby \\ MATFORSK, The Norwegian Food Research Institute \\ Osloveien 1, NO-1430 As, Norway
}

\begin{abstract}
Dairy products contain natural occurrences of porphyrins, which are active and very effective photosensitizers. The origin and formation of these tetrapyrrols are yet unclear, and the concentration levels are not known. In this study, the approximate concentrations of protoporphyrin IX and haematoporphyrin in butter were determined by the use of non-destructive front face fluorescence spectroscopy. The concentrations for both compounds were in the region $0.02-0.03 \mathrm{ppm}$.
\end{abstract}

KEY WORDS: porphyrins, butter, fluorescence spectroscopy, photooxidation

\section{INTRODUCTION}

Dairy products are in general susceptible to photooxidation due to natural contents of photosensitizers. To develop storage conditions providing maximal protection, it is necessary to have detailed knowledge of the presence and properties of the photosensitizers in the product (Skibsted, 2000).

Riboflavin has been regarded as the active photosensitizer in dairy products (Borlet et al., 2001). Recent results, however, have demonstrated that dairy products have natural contents of effective photosensitizers acting in the visible spectrum, such as protoporphyrin and chlorophyll-like molecules (Wold et al., 2005). This means that photooxidation in dairy products is caused not only by radiation in the UV - blue region, but also by light in the green-red region.

Presently, we assume that there are at least six different light sensitizers present in dairy products: riboflavin, protoporphyrin, haematoporphyrin, a chlorophyll

\footnotetext{
* Supported by the Norwegian Research Council

${ }^{1}$ Corresponding author: e-mail: jens.petter.wold@matforsk.no
} 
$a$-like derivative, and two unidentified tetrapyrroles, probably porphyrins (Wold et al., 2006). The origin and formation of these tetrapyrroles are yet unclear, and the concentration levels are not known. We do, however, know that the concentrations vary from product to product and between different production batches of the same product.

The natural occurrence of porphyrins and chlorins in dairy products was discovered by the use of front face fluorescence spectroscopy. This method enables rapid, non-destructive and simultaneous measurement of riboflavin, porphyrins, and chlorophylls. In this study we have used this method to perform an approximate quantification of protoporphyrin and haematoporphyrin in butter.

\section{MATERIAL AND METHODS}

\section{Materials}

Packages of $500 \mathrm{~g}$ from the same batch of dairy butter (Tine Smør) were obtained from TINE BA, Norway. Butter directly from the cooler was placed in glass beakers and weighed to batches of $80 \mathrm{~g}$. Before adding the pure chemicals the butter was tempered to approximately $25^{\circ} \mathrm{C}$. At this temperature the butter got a smooth consistence and was easy to stir by hand.

The pure chemicals protoporphyrin IX $\left(\mathrm{C}_{34} \mathrm{H}_{34} \mathrm{~N}_{4} \mathrm{O}_{4}, 95 \%\right.$, Sigma CAS 55312-8) and haematoporphyrin $\left(\mathrm{C}_{34} \mathrm{H}_{38} \mathrm{~N}_{4} \mathrm{O}_{6}, 50 \%\right.$, Sigma CAS 14459-29-1) were obtained from Sigma Aldrich Chemie GmbH (Steinheim, Germany).

\section{Design, treatments and measurements}

The pure compounds were weighed out in small aluminium vessels by use of a precision balance with readability of $0.0001 \mathrm{~g}$. Two stock butters containing each of the two compounds were made, and held the concentrations $19 \mathrm{ppm}$ and 11 $\mathrm{ppm}$ for protoporphyrin IX and haematoporphyrin, respectively. The appropriate amount of stock butter was added to the $80 \mathrm{~g}$ butter batches to obtain a reasonable span in the added concentrations, typically from about 0.05 to $0.4 \mathrm{ppm}$. The stock butter was hand stirred into the butter. Stirring was stopped when no visual particles of the added compound were left. The result was a smooth paste with even colour. Each sample was put in a cuvette, cooled to $4^{\circ} \mathrm{C}$ and stored in the dark before fluorescence measurements.

Front face fluorescence emission spectra in the range $500-730 \mathrm{~nm}$ for excitation wavelength $380 \mathrm{~nm}$, were measured directly on the surface of the butter samples. An optical bench system was used, previously described by Wold et al. (2005). Two parallels of each sample were measured. 


\section{Data analysis}

A fluorescence spectrum from a butter sample without added compounds were subtracted from the spectra from spiked samples. The peak values for protoporphyrin and haematoporphyrin were then measured at the wavlengths 635 and $620 \mathrm{~nm}$, respectively, for each concentration. A linear regression between these peak values and the known concentrations were made by least squares regression. Based on the regression line, the original intrinsic concentrations could be estimated.

\section{RESULTS}

The actual concentrations of added compounds were $0.047,0.10,0.21$, and $0.41 \mathrm{ppm}$ for protoporphyrin IX, and $0.025,0.05,0.10,0.20,0.30$, and 0.41 for haematoporphyrin.
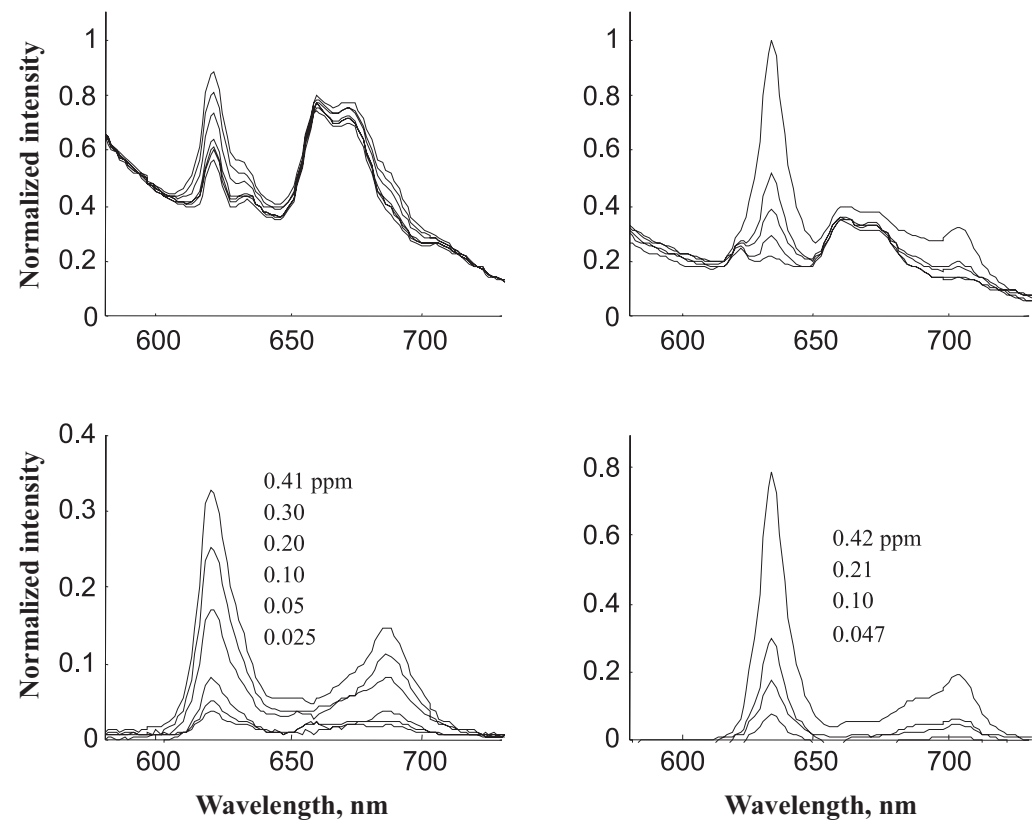

Figure 1. Upper panel: Fluorescence spectra from haematoporphyrin (left) and protoporphyrin (right) spiked samples and butter reference. Under: Spectra after subtraction of butter reference

Figure 1 shows fluorescence spectra of pure butter, and butter spiked with either protoporphyrin IX or haematoporphyrin. Spectra after subtraction of the spectrum from pure butter are also shown, and resemble spectra from the pure compounds. 
A systematic increase in peak height was observed with increasing concentration. Note that the original concentrations of the compounds were considerably less than the added concentrations.

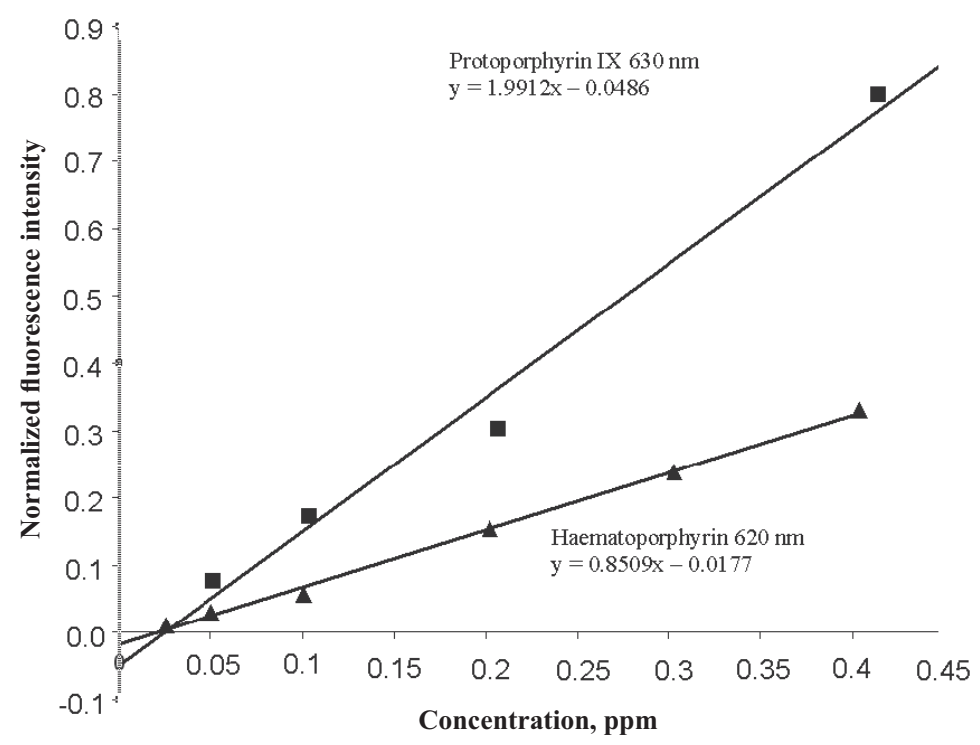

Figure 2. Regression lines for concentrations versus fluorescence intensity

Figure 2 shows regression lines obtained for protoporphyrin IX and haematoporphyrin. These lines intersect the $\mathrm{x}$-axis at $0.024 \mathrm{ppm}$ and at $0.020 \mathrm{ppm}$ for protoporphyrin IX and haematoporphyrin, respectively. These intersections indicate the natural contents of these compounds.

\section{DISCUSSION}

The results indicate that the concentrations of protoporphyrin IX and haematoporphyrin in butter are in the $0.02-0.03 \mathrm{ppm}$ region. The exact concentrations in butter vary from batch to batch, and probably from season to season. There may be errors related to the experimental procedures. Thus, the blends were probably not perfectly homogeneous, and some inaccuracies in weighting and measurement may have occurred. Previous fluorescence measurements (Wold et al., 2005) suggest that butter has higher concentrations of porphyrins than other dairy products, such as milk, cheese and sour cream. It is noteworthy that photosensitizers in such low concentrations seem to have a major impact on photooxidation of butter and cheese, as shown by Wold et al. $(2005,2006)$. 


\section{CONCLUSIONS}

The concentrations of protoporphyrin IX and haematoporphyrin in butter are in the range of 0.02-0.03 ppm. In spite of these low concentrations, they can be quantified rapidly and non-destructively by front face fluorescence spectroscopy, and may have impact on the photostability of the product.

\section{REFERENCES}

Borlet F., Sieber R., Bosset J.O., 2001. Photo-oxidation and photoprotection of foods, with particular reference to dairy products. An update of a review article (1993-2000). Sci. Aliment. 21, 571590

Skibsted L.H., 2000. Light-induced changes in dairy products. Bull. Int. Dairy Fed. 346, 4-9

Wold J.P., Bro R., Veberg A., Lundby F., Nilsen A.N., Moan J., 2006. Active photosensitizers in butter detected by fluorescence spectroscopy and multivariate curve resolution. J. Agr. Food Chem. 54 (26), 10197-10204

Wold J.P., Veberg A., Nilsen A.N., Juzenas P., Iani V., Moan J., 2005. The role of naturally occurring chlorophyll and porphyrins in light induced oxidation of dairy products. A study based on fluorescence spectroscopy and sensory analysis. Int. Dairy J. 15, 343-353 